\title{
Plattenosteosynthese von dorsal
}

\author{
Hartmut R. Siebert
}

\section{Zusammenfassung:}

Aufgrund der hohen Morbidität gerade nach dorsaler Plattenosteosynthese mit 3,5-mm-Platten und der Entwicklung neuer winkelstabiler Platten ist heute die Indikation zur dorsalen Plattenosteosynthese nur wenigen Fällen vorbehalten (C 2, C 3-Frakturen mit disloziertem ulnaren Kantenfragment („Schlüsselfragment“!). Der Zugangsweg dorsal für größere Implantate erfordert die Eröffnung des 3. und 4. Strecksehnenfaches. Das Anlegen einer 3,5-mm-TPlatte macht das Abtragen des Tuberculum listeri notwendig, der direkte
Kontakt zwischen Platte und Sehnen kann durch eine sogenannte Retinaculumplastik vermindert werden und verhindert damit anhaltende Sehnenreizungen, die zu einer frühzeitigen Metallentfernung führen. Unter Berücksichtigung der biomechanischen Prinzipien des 3-Säulen-Modells werden anstelle groß dimensionierter Platten kleinere Abstützplatten der Dimension 2,0 bis $2,7 \mathrm{~mm}$ in der sogenannten Doppelplatten-Technik durchgeführt. Kleinere Zugänge und dünnere Profile senken erheblich das Risiko einer Wundheilungsstörung oder chronischen Sehnenreizung. mit den Sehnenscheiden fest verbunden, so dass eine Trennung dieser Schichten bei der chirurgischen Präparation nicht möglich ist. Somit muss zum Anlegen einer flachen T-Platte Dimension 3,5 $\mathrm{mm}$ das Tuberculum listeri abgetragen und die Strecksehnen aus ihren Fächern über die Platte verlagert werden. Um die Sehnenreizung, hervorgerufen durch den direkten Kontakt mit dem TSchenkel der Platte, zu minimieren, wird deshalb die Technik der so genannten Retinaculumplastik angewandt, bei der ein Teil des Retinaculum extensorum zwischen Platte und Sehnen verlagert wird (Abb.2c). Trotz dieser plastischen Maßnahme findet sich bei Durchführung der dorsalen Plattenosteosynthese mit der 3,5-mm-T-Platte eine recht hohe Rate von chronischen Sehnenreizungen

\section{Einleitung}

Plattenosteosynthesen am distalen Speichenende können palmar wie dorsal durchgeführt werden. Biomechanisches Prinzip ist die Abstützung der metaphysären oder artikulären Kantenfragmente und die Neutralisation von Rotationskräften bei der Anwendung von interfragmentären Zugschrauben.

Während die palmare Plattenlage am distalen Radius aufgrund der flachen bis leicht konkaven Fläche des distalen Radius ideal für das Anlegen einer vorgeformten T-Platte ist und Sehnen und Nerven durch die Muskelschicht des Musculus pronator quadratus getrennt sind, ist dorsal das Speichenende nicht flach, sondern eher zeltförmig. Die Strecksehnen verlaufen in mehr oder weniger ausgeprägten Rinnen im Knochen und das Periost ist
OP-JOURNAL 2003; 19: 34-40

(c) Georg Thieme Verlag Stuttgart · New York

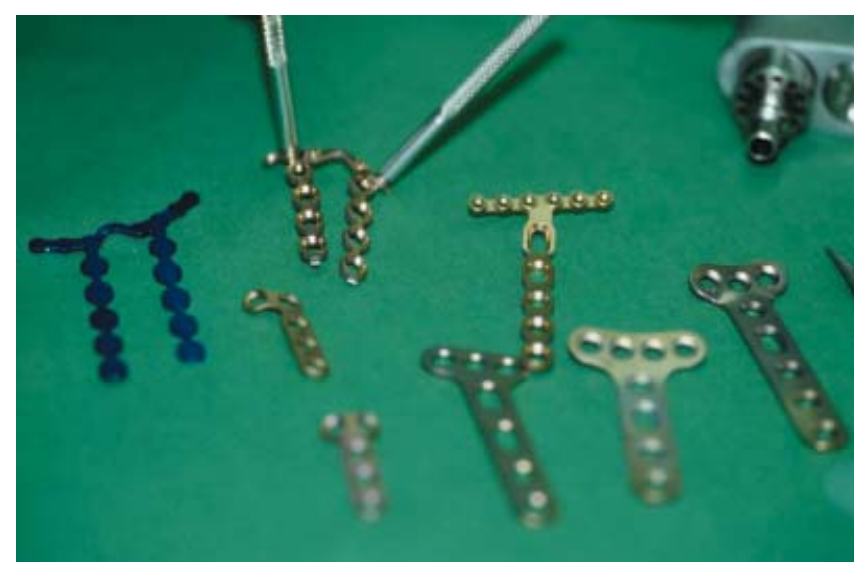

Abb.1 a T-Abstützplatten $3,5 \mathrm{~mm}$, 2,7-mm-Gliederplatte und 2,0- und 2,7-mm-Form-Platten.

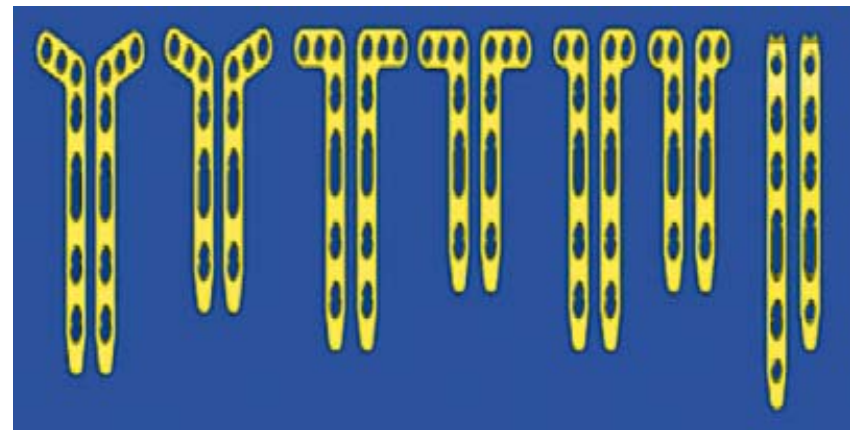

Abb. 1 b $\quad 2,4 \mathrm{~mm}$ winkelstabile dorsale Formplatten. 


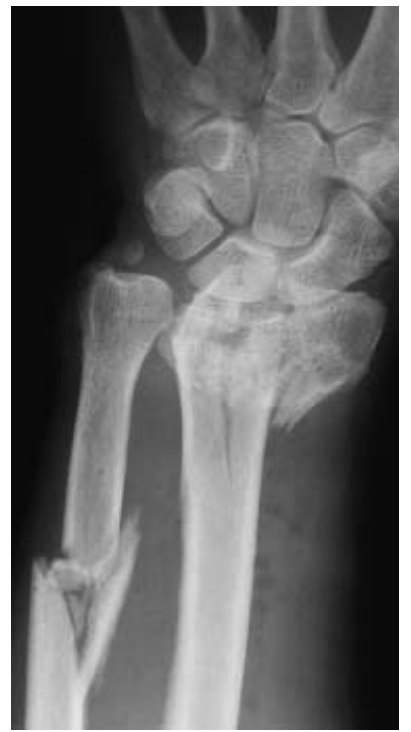

Abb. 2a Distale Radiusfraktur Typ C 2/3, Ulnaschaftfraktur Röntgen a.p. präop.
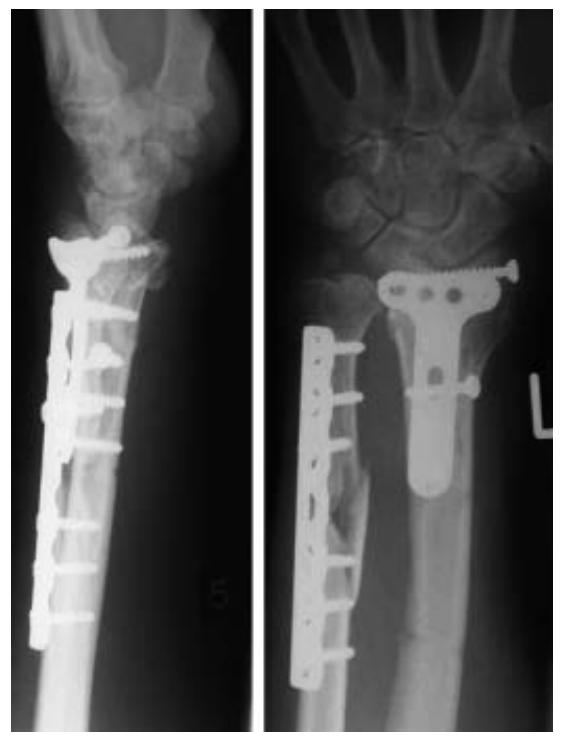

Abb.2b Röntgen nach Versorgung mit 3,5-mm-T-Platte dorsal und freier Zugschraube.

bis hin zu Sehnenrupturen. Zusätzlich unterstützt der ausgedehnte Zugangsweg mit Mobilisierung des 3., 4. und 2. Strecksehnenfaches die Rate an postoperativen Wundheilungsstörungen.

Ziel der Platten-Osteosynthese: Sichere Fixation, frühe Übungsstabilität, geringe perioperative Morbidität.

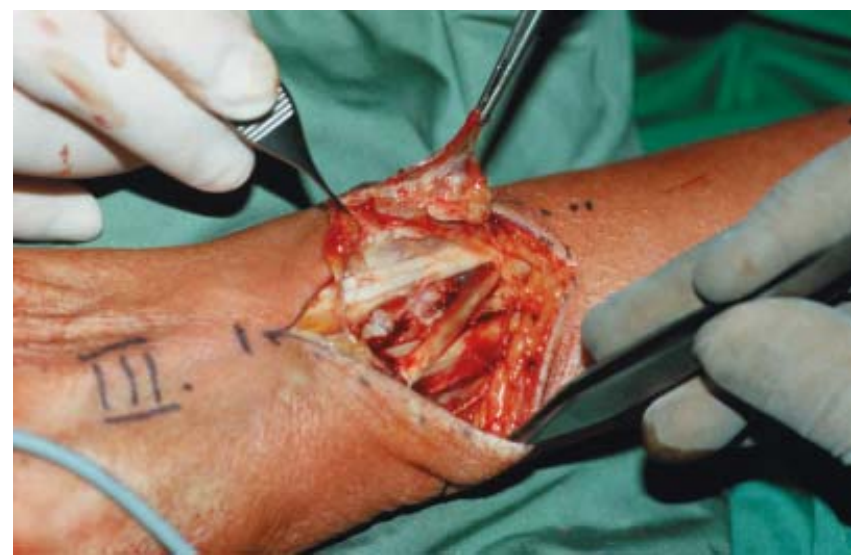

Abb. 2c Op-Situs dorsaler Zugang, Bildung von Retinaculum-Lappen, Freilegen des 3. und 4. Strecksehnenfaches.

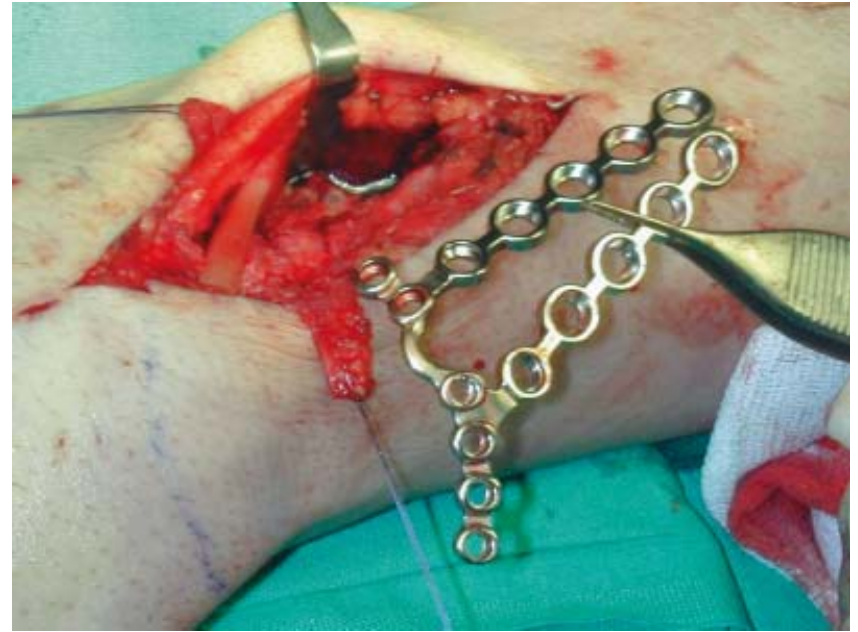

Abb. 3a Erweiterter dorsaler Zugang, Anbringen Phi-Platte. Beachte: Retinaculum-Lappen angeschlungen.
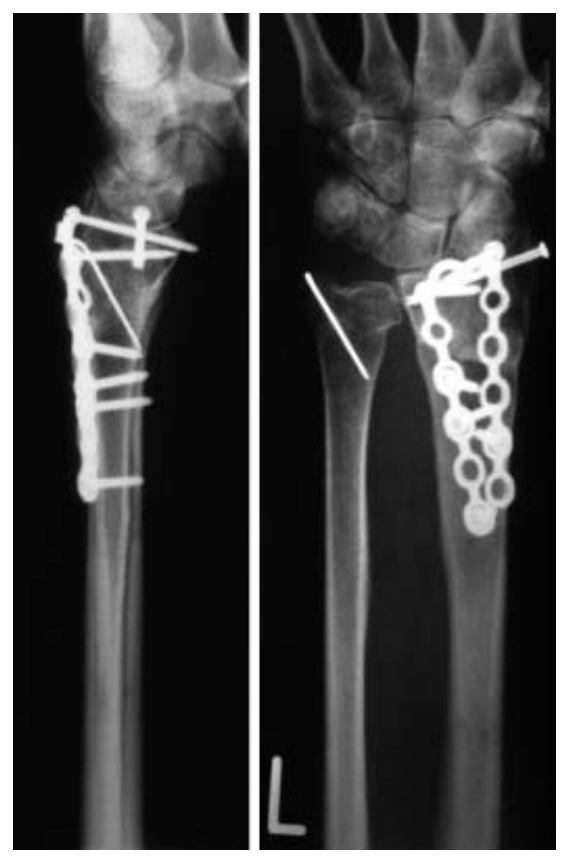

Abb. 3b Röntgenbild - a.p., seitlich - einer mit dorsaler Phi-Glieder-Platte versorgten C 2 Fraktur.

\section{Neue Implantat-Entwicklungen}

Die bei der 3,5-mm-T-Abstützplatte häufig beobachteten Wundheilungsstörungen und chronischen Sehnenirritationen förderten die Entwicklung kleiner dimensionierten Platten für das dorsale distale Speichenende (Abb.1 $\mathbf{a}$ u.b)

Deutlich erhöhtes Risiko der Wundheilungsstörung und Sehnenirritation bei dorsaler Plattenlage $3,5 \mathrm{~mm}$

Verringerung der Morbidität durch limitierte Zugänge, Kombinationsosteosynthesen z.B. mit perkutan gelegten Zugschrauben, klein dimensionierten Abstützplatten 2,0 bis $2,7 \mathrm{~mm}$.

\section{2,7-mm-Gliederplatte Phi-Platte}

Die zweischenkelige kleine Gliederplatte - 2,7 mm - mit distalen eingliedrigen Armen zur anatomischen Anpassung und Umfassung des Processus styloideus radii sowie der distalen ulnaren Kanten war die vorübergehende Antwort auf die Komplikationen bei der Anwendung von 3,5-mm-T-Platten dorsal. Auf die Abtragung des zusätzlich stabilisierenden 


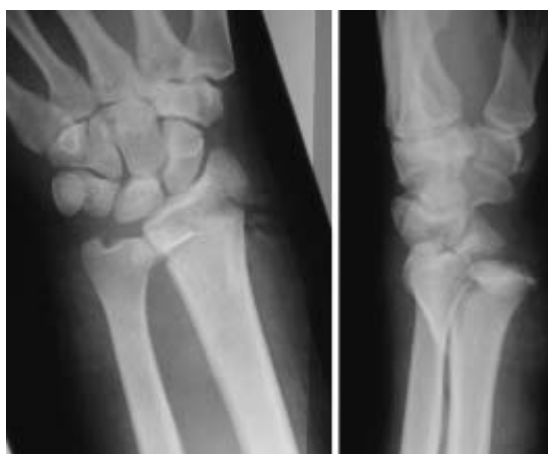

Abb.4a Röntgenbild - a.p., seitlich - C 2Fraktur präoperativ.
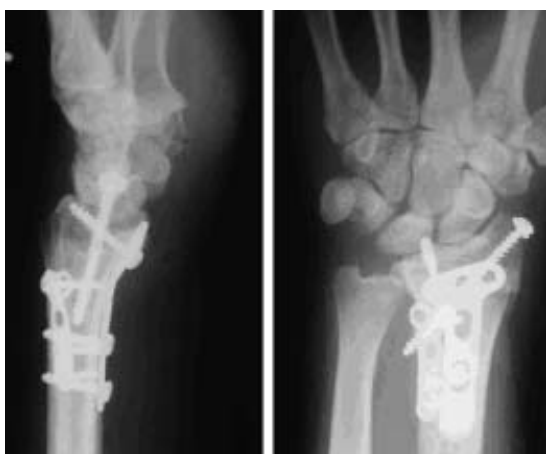

Abb. 4b Röntgenbild in 2 Eb. postoperativ. 2 2,0 mm palmar und dorsal angelegte Platten mit freier Zugschraube. „Sandwich-Technik“.

und die Sehne des Extensor pollicis longus führenden Tuberculum listeri kann verzichtet werden. $1,8 \mathrm{~mm}$ winkelstabile Bolzen unterstützen die Verankerung der distalen Gliederarme bei osteoporotischem Knochen und ermöglichen auch kleineren Fragmente bei intraartikulären C 2-Frakturen sicher zu stabilisieren. Die 2 langen, nach proximal reichenden Schenkel der Gliederplatte können entsprechend des individuellen Frakturmusters zurechtgeschnitten werden. Neben den $1,8 \mathrm{~mm}$ winkelstabilen Bolzen werden die langen Schenkel der Gliederplatte im metaphysären Bereich mit 2,7-mmKortikalisschrauben fixiert. Trotz dieser Verbesserungen ist ein relativ großer dorsaler Zugang mit Freilegung der Strecksehnen zur anatomiegerechten Anbringung erforderlich. Auch das Problem des direkten Kontaktes der Strecksehnen mit dem Implantat wird nicht ganz ausgeräumt (Abb. $\mathbf{3}$ b u. c).

\section{Doppelplatten-Osteosynthesen $(2,0,2,4$, und $2,7 \mathrm{~mm})$}

Während die Versorgung extraartikulärer metaphysärer Frakturen mit den verschiedensten Implantaten sicher und komplikationsarm von palmar durchge- führt werden kann, bleibt bei den intraartikulären Frakturen das Problem der anatomiegerechten Einrichtung und Fixierung des dorso ulnaren Schlüsselfragmentes. Häufig muss das dorso ulnare Kantenfragment mit seinen distal und ulnar tragenden Gelenkanteilen (radiokarpal+radioulnare Gelenkflächen!) offen reponiert werden. Die Fixation mit Kirschner-Drähten oder Zugschrauben bietet nicht immer die erforderliche Übungsstabilität, so dass die Anwendung von klein dimensionierten (2,0 oder $2,7 \mathrm{~mm}$ ) Form-Platten über einen direkten Zugang vom 3.Strecksehnenfach aus eine Lösung dieses Problems darstellt. Daneben kann das radiale Hauptfragment mit dem Processus styloideus radii und das intermediäre Fragment mit der Gelenkfläche der Fossa lunata durch eine zusätzlich radio dorsal eingebrachte, ebenso klein dimensionierte Platte fixiert werden. Der Vorteil dieser so genannten Doppelplatten-Osteosynthese-Technik liegt in der niedrigeren perioperativen Morbidität des Zuganges. Wir führen diese Doppelplatten-Osteosynthese häufig dann durch, wenn bei C 2- und C 3Frakturen primär ein gelenküberbrückender Fixateur externe angelegt wurde und in einer 2.Sitzung die anatomische Gelenkflächenrekonstruktion erforderlich wird. Gelegentlich wird auch die Doppelplatten-Osteosynthese in Form der sogenannten „Sandwich-Technik“ notwendig, um bei palmarer Plattenlage das dorsale Kantenfragment mit einer kleinen 2,0-T- oder L-Platte abzustützen (Abb.4a-c, 5 a-e) $[1,3,4]$.

Die Doppelplattenosteosynthese verringert aufgrund der geringeren Zugangsmorbidität postoperative Wundheilungsstörungen und chronische Sehnenreizungen. Sie ist indiziert bei bestimmten intraartikulären Frakturtypen, häufig nach primärer Fixateur externe Versorgung.

Bei osteoporotischen Frakturen bleibt die Verankerung der 3,5 oder 2,0 bis $2,7 \mathrm{~mm}$ Schraubenimplantate problematisch, so dass hier manchmal mit Schraubenlockerung und sekundärer Dislokation zu rechnen ist. Abhilfe schaffen hier:

- Die Defektauffüllung mit körpereigenem Knochenmaterial oder

- Knochenersatzmaterialen keramischer oder Kunststoffart

- winkelstabile Plattenimplantate

\section{2,4 mm winkelstabile Formplatten}

Das Prinzip winkelstabiler Platten wurde in den letzten 2 Jahren auch auf die Form-

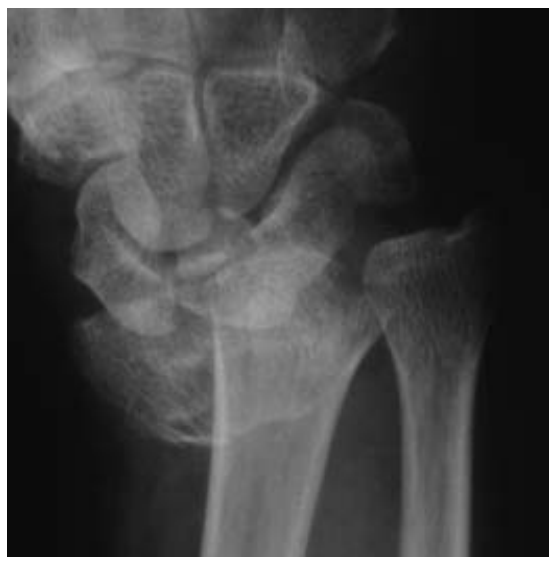

Abb.5a Röntgenbild dislozierte C 3-Fraktur a.p.

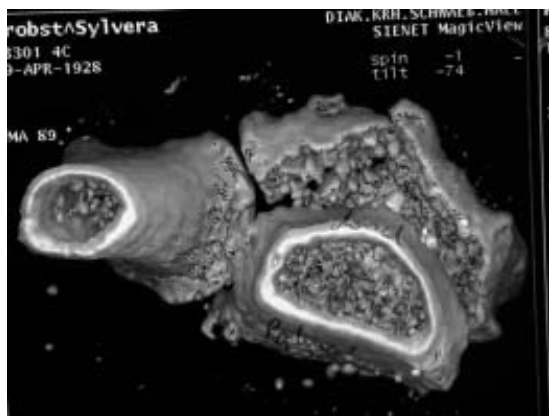

Abb.5b Dreidimensionale CT-Darstellung. Fall Abb. 5a. Beachte: Ulnares Kantenfragment dorsal disloziert.

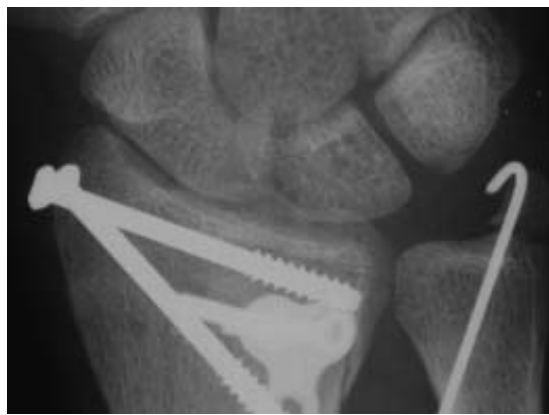

Abb.5c Röntgenbild a.p. Vergrößerung: Zst. n. geschlossener Reposition, Fixateur externe und limitiertem Zugang mit dorsaler ulnarer 2,0-Abstützplatte und freien Zugschrauben vom Processus styloideus radii, Fixation Processus styloideus ulnae mit Kirschner-Draht. 

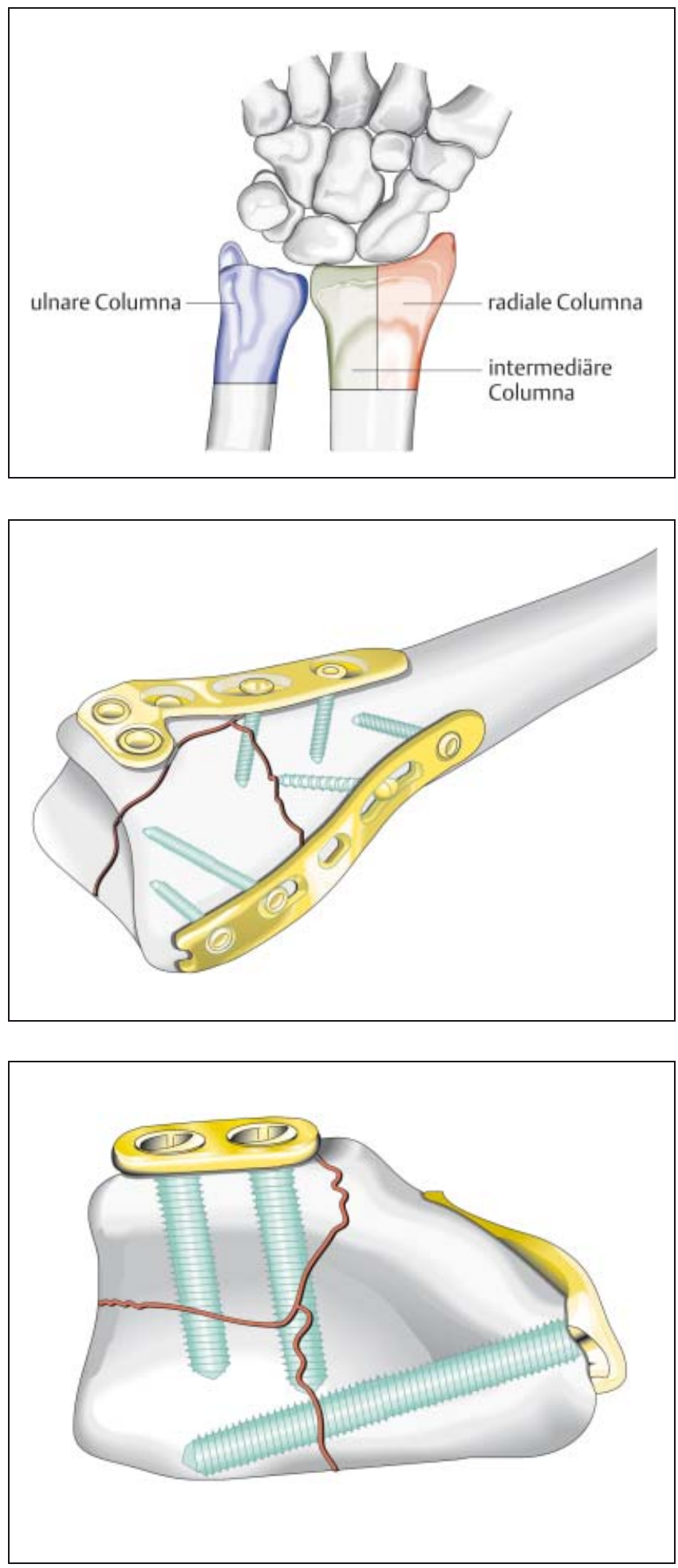

Abb.6a 3-SäulenModell nach D. Rikli.

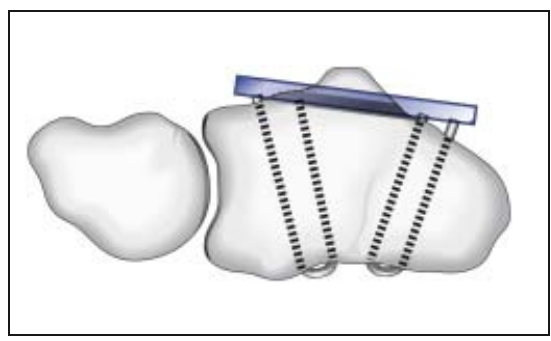

Abb.6b Dorsale Plattenlage 3,5 mm mit Abtragung Tuberculum listeri nach R. Widmann/ D. Rikli.

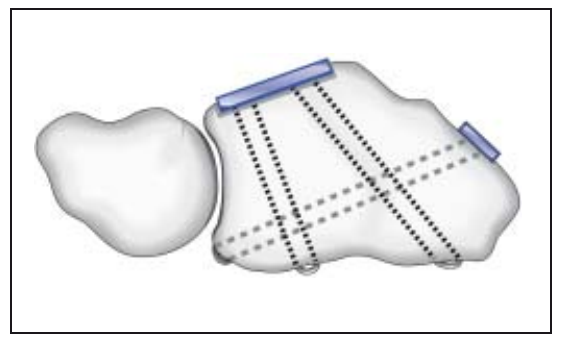

Abb.6c Dorsale Doppelplattenlage 2,4 mm nach D. Rikli/R. Widmann.

platten zur Versorgung distaler Speichenbrüche angewendet. So stehen 3,5-mmAbstützplatten als winkelstabile Implantate für den palmaren Zugang zur Verfügung und werden mit großem Erfolg bei den verschiedensten Frakturtypen am distalen Speichenende angewendet.

Abb.6e Doppelplattenlage von distal gesehen. Beachte: Radiale und dorso-ulnare Lage der 2,4-mm-platten nach $D$. Rikli/R. Widmann. Rikli und Regazzoni. Diesem Modell lie-
Die Schaffung spezieller dorsal anzubringender winkelstabiler Platten folgte dem biomechanischen 3-Säulen-Prinzip von gen biomechanische Analysen der Lastverteilung am distalen Vorderarm zugrunde. Dabei wird

1. eine radiale Säule, bestehend aus dem Processus styloideus radii und der Gelenkfläche zum Kahnbein,

2. der intermediären Säule, bestehend aus dem ulnaren Anteil des Radius mit seiner Gelenkfläche zum Lunatum und zur Ulna und

3. der ulnaren Säule, bestehend aus dem Ulnakopf, dem ulnaren Anteil des distalen Radioulnargelenkes und dem so genannten ulnokarpalen Bandkomplex mit dem Discus triangularis, seinen Bandstrukturen und der Sehne des Extensor carpi ulnaris beschrieben (Abb.6a) [2, 3].

Bei einer Fraktur des distalen Speichenendes sollen alle 3 Säulen, so betroffen und disloziert, eingerichtet und stabilisiert werden. Dabei wird die radiale Säule mit einer radial platzierten Abstützplatte, die intermediäre Säule mit einer dorso- 


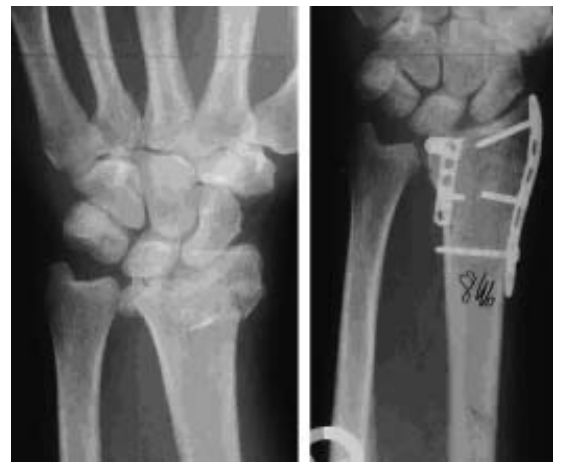

Abb. 7a Röntgenbild a.p: Vor und nach Versorgung einer $C 2$ Fraktur in dorsaler Doppelplatten-Technik 2-4 mm LC-Platten.
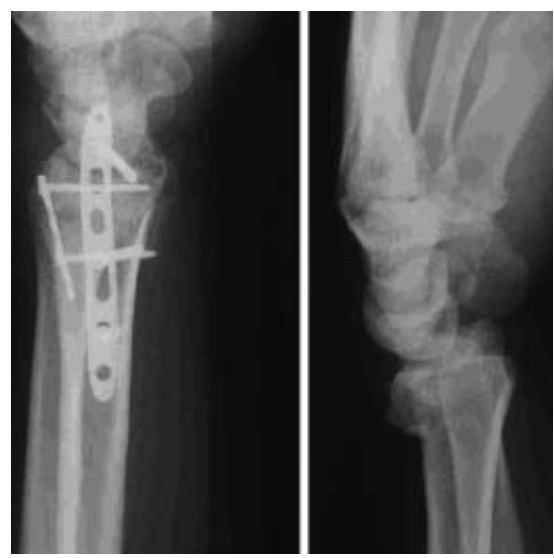

Abb.7b Röntgen-Bilder seitlich: Vor und nach Versorgung mit 2,4 mm dorsaler Doppelplatten-Technik.

ulnar platzierten Platte abgestützt. Sie kontrolliert das dorso-ulnare Schlüsselfragment. Je nach dem Grad der ulnaren Instabilität muss auch die ulnare Säule stabilisiert werden, beispielsweise bei einem basisnahen dislozierten Abriss des Processus styloideus ulnae $(2,3)$.

Ein Set der winkelstabilen Formplatten 2,4 mm - aus Titan wurde von der Firma Mathys, Bettlach, Schweiz, unter der Leitung von M. Widmann und in Zusammenarbeit mit einer Gruppe von Chirurgen der AO (J. B. Jupiter, Boston; M. Plekko, Innsbruck; G. Laurie, Florenz; D. A. Rikli, Luzern; H. R. Siebert, Schwäb. Hall) entwickelt. Für die intermediäre Säule und damit Stabilisierung der dorso-ulnaren Fragmente stehen L-Platten, für die radiale Säule eine S-Platte sowie für die palmare Anwendung eine T-Platte zur Verfügung. Sämtliche Plattenlöcher können mit winkelstabilen Schrauben besetzt werden $(\mathbf{A b b . 6 b}, \mathbf{c})(\mathbf{A b b} \mathbf{7} \mathbf{a}-\mathbf{e})$. Die palmare Platte wird nach dem Konzept der Abstützplatte und Dank der winkelstabilen Schrauben, auch nach dem

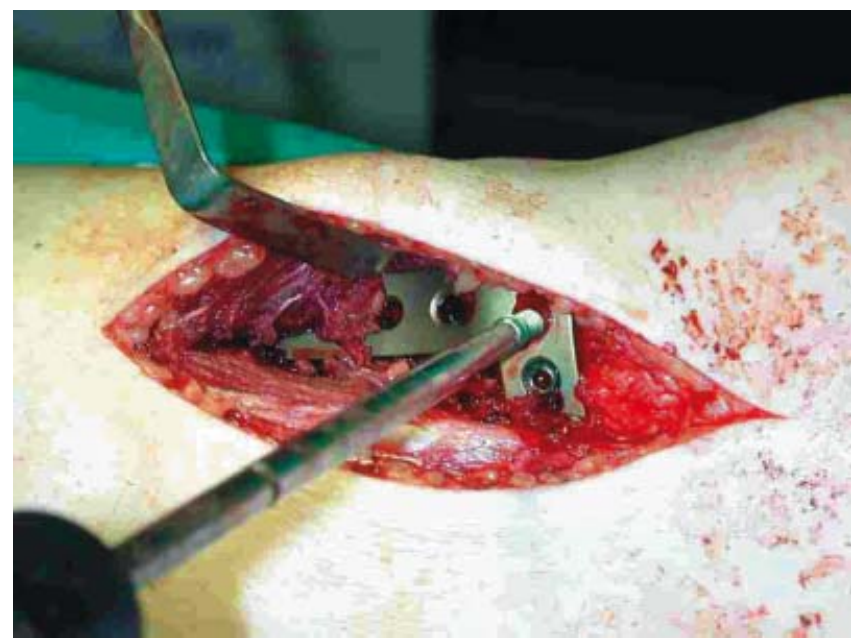

Abb. 7c Op-Situs, ulno dorsale Lage der L-Abstützplatte 2,4 mm. Beachte: Erhaltenes Tuberculum listeri.

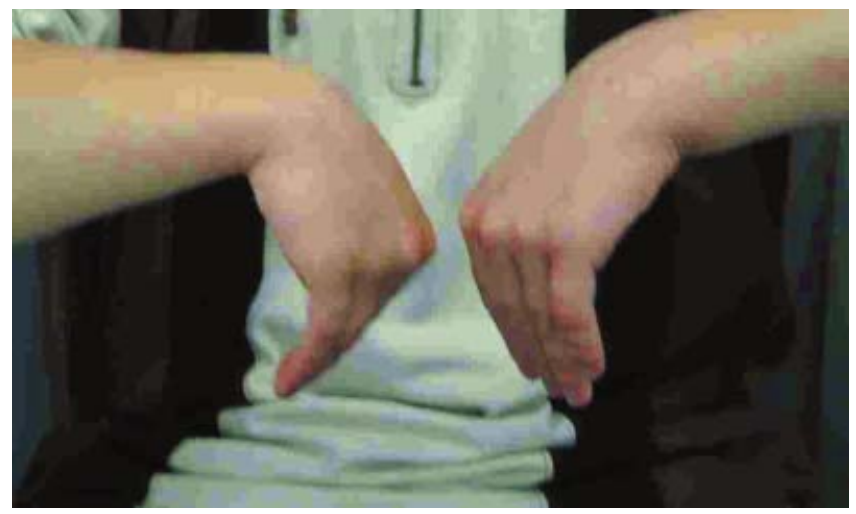

Abb. 7d Funktionsaufnahmen des Patienten 6 Wochen postoperativ. Im Seitenvergleich Funktion Palmarflexion. Operierte Seite li.

Tab. 1 Komplikationen nach dorsaler Plattenosteosynthese (eigene Ergebnisse)

\begin{tabular}{llllll}
\hline A 1993 mit & 3,5-mm-T-Platten & & \\
\hline $\begin{array}{l}\text { Anzahl } \\
\text { Patienten }\end{array}$ & $\begin{array}{l}\text { Wundhei- } \\
\text { lungsstörung } \\
\text { 2.Op. erfor- } \\
\text { derlich }\end{array}$ & $\begin{array}{l}\text { Sehnenprob- } \\
\text { lem ME } \\
\text { vorgezogen }\end{array}$ & Sehnenruptur & $\begin{array}{l}\text { komplexes } \\
\text { regionales } \\
\text { Schmerz- } \\
\text { syndrom }\end{array}$ & $\begin{array}{l}\text { N. radialis } \\
\text { Läsion }\end{array}$ \\
\hline 52 & 3 & 10 & 1 & 2 \\
\hline B 2001/2002 & mit 2,4 mm winkelstabilen DR-Platten & 1 & \\
\hline $\begin{array}{l}\text { Anzahl } \\
\text { Patienten }\end{array}$ & $\begin{array}{l}\text { Wundhei- } \\
\text { lungsstörung } \\
\text { 2. Op. erfor- } \\
\text { derlich }\end{array}$ & $\begin{array}{l}\text { Sehnenprob- } \\
\text { lem ME } \\
\text { vorgezogen }\end{array}$ & Sehnenruptur & $\begin{array}{l}\text { komplexes } \\
\text { regionales } \\
\text { Schmerz- }\end{array}$ & $\begin{array}{l}\text { Näradialis } \\
\text { Läsion }\end{array}$ \\
\hline 32 & 1 & 0 & 0 & 0 & 0 \\
\hline
\end{tabular}

Konzept der Winkelplatte, angewendet und ist insbesondere für kleinere Kantenfragmente der größer dimensionierten 3,5 mm winkelstabilen Platte überlegen. Die bislang vorliegenden Ergebnisse der Anwendung der $2,4 \mathrm{~mm}$ winkelstabilen Formplatten dorsal zeigen gute Ergebnisse mit einer deutlich geringeren Mor- bidität und Folgeschäden wie der chronischen Sehnenreizung (Tab.1).

\section{Chirurgischer Zugang bei Anwendung} der 2,7- und 2,4-mm-Platten

Eine Längsinzision von 5 bis $6 \mathrm{~cm}$ über dem Tuberculum listeri mit Abschieben 


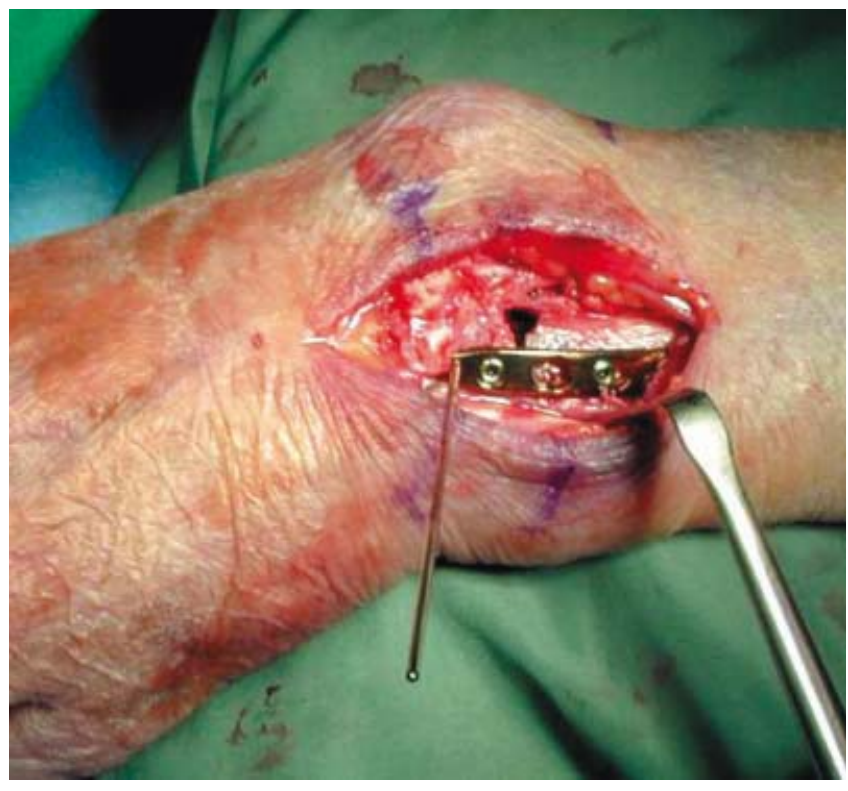

Abb. 8a Op-Situs, radial angelegte 2,4mm-Platte, Fixation temporär mit Kirschner-Draht. Ausgeprägte Osteoporose, 84-jährige Pat.
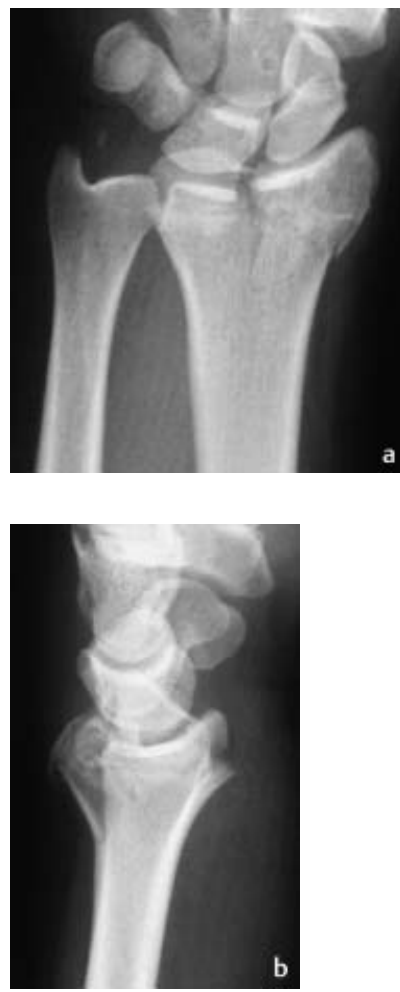

Abb. 9a, b: Röntgenbilder präoperativ $C 3$ Fraktur, Beachte: disloziertes ulnares Kantenfragment dorsal.
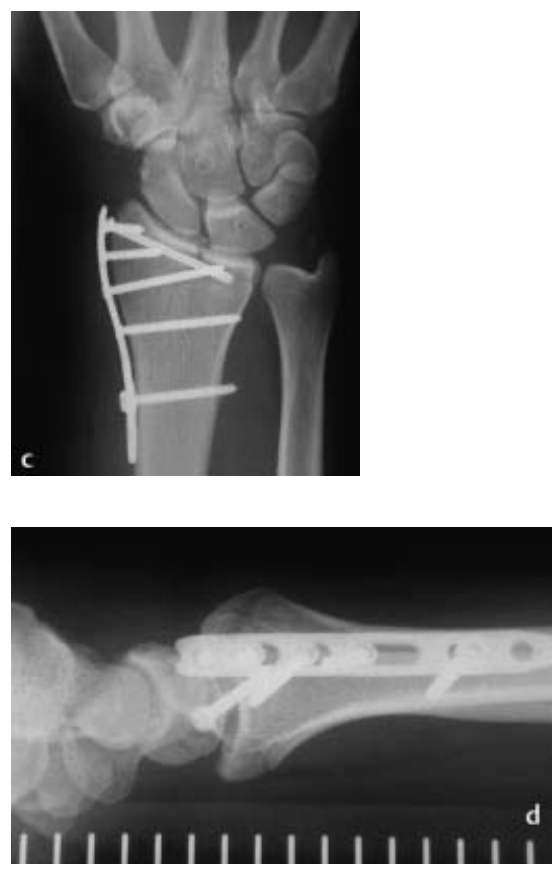

Abb.9c,d: Röntgenbilder a.p. und seitlich 6 Wochen nach Versorgung mit radial angelegter $2,4 \mathrm{~mm}$ winkelstabiler Platte. Das ulnare dislozierte Kantenfragment wurde mit einer von radial eingebrachten $3,0 \mathrm{~mm}$ kanülierten Schraube zusätzlich fixiert. tes radialen Pfeilers (Abb. 8b). Der Rückzug nach Durchführung der Plattenosteosynthese schließt den Verschluss des 3. Strecksehnenfaches unter der

\section{Indikation für die dorsale} Plattenosteosynthese

1. Dorsal dislozierte intraartikuläre Mehrfragmentfrakturen, insbesondere mit dem dorso-ulnaren Kantenfragment Typ AO C 2, C 3

2. Metaphysäre Defekt- und Trümmerfraktur mit Einstrahlung von Frakturausläufern in das Gelenk und zusätzlich karpalen Verletzungen

3. Dorsale offene Frakturen der distalen Speichenbasis d,e)

Die dorsale Plattenosteosynthese mit einer $3,5 \mathrm{~mm}$ dimensionierten Abstützplatte ist Einzelfällen vorbehalten. Bei den meisten Frakturtypen ist heute mit winkelstabilen Implantaten eine anatomische Reposition und Fixation durch eine palmar angelegte Platte möglich.

Besonderheiten der Plattenosteosynthese bei Osteoporose

Die Anwendung der Plattenosteosynthese bei osteoporotisch verändertem Knochengewebe führt häufig zu einer sekundären Dislokation und Lockerung oder/und der Notwendigkeit einer Defektauffüllung mit körpereigenem Knochengewebe oder Knochenersatzmate- 
rialien. Mit der Einführung winkelstabiler Platten kann vielfach auf die metaphysäre Defektauffüllung verzichtet werden. Der Schraubenhalt im osteoporotischen Knochengewebe ist aufgrund des Verriegelungssystems Schraube/Platte deutlich erhöht. Bei kleineren intraartikulären Fragmenten bietet die kleiner dimensionierte $2,4 \mathrm{~mm}$ winkelstabile Platte den Vorteil einer sicheren Fixation der Fragmente.

Unser Vorgehen bei intraartikulären Mehrfragmentfrakturen und/oder HochRasanz-Traumen des distalen Speichenendes und Handgelenkes

In diesen Situationen wählen wir gerne den zweizeitigen Weg. Am Unfalltag geschlossene Reposition, gelenküberbrückender Fixateur externe. Nach 2 bis 5 Tagen und eventuell weiterführender bildgebender Diagnostik - z.B. CT - führen wir dann die definitive Versorgung mit einer dem Verletzungsmuster angepassten internen oder kombinierten internen/externen Stabilisierung durch.

Vorteil dieses zweizeitigen Vorgehens ist die sichere Erfassung des Verletzungsmusters, auch mit aufwändigen bildgebenden Verfahren, die anatomische Wiederherstellung der Gelenkfläche durch, limitierte, direkte Zugänge palmar wie dorsal und die Durchführung dieses Eingriffes zu optimalsten äußeren Bedingungen. Meistens kann der gelenküberbrückende Fixateur externe nach interner Stabilisierung entfernt werden.

Dieses Vorgehen ermöglicht ein minimal invasives Vorgehen und reduziert die Trage-/Liegezeit des gelenküberbrückenden Fixateur externe.
Zusatzverletzungen bei distalen Speichenbrüchen

Verletzungen des interkarpalen (scapholunären, ulnotriquetralen) Bandapparates, der discoligamentären Strukturen des ulnocarpalen Komplexes, Frakturen des distalen Ellengriffels und Handwurzel-Frakturen (häufig: Kahnbeinfrakturen) bedürfen der speziellen Diagnostik und Behandlung. Neben der aufwändigen arthroskopischen Diagnostik erlaubt die z.B. intraoperative, vor Op-Ende durchgeführte kinematographische Untersuchung des Handgelenkes nach stabiler osteosynthetischer Versorgung die Erkennung instabiler Begleitverletzungsfolgen.

Bei bestimmten typischen Frakturmustern ist deshalb die Bildwandler-Untersuchung nach Abschluss der Osteosynthese mit geführten Bewegungen des Handgelenkes zur Erkennung einer SL-Instabilität höheren Ausmaßes und des Vorliegens einer Instabilität im distalen Radioulnargelenk und ulno-carpalen Komplexes unerlässlich.

\section{Schlussfolgerung}

Die Plattenosteosynthese am distalen Speichenende ist aufgrund der besonderen dorsalen anatomischen Verhältnisse des körperfernen Radius für die Anlage einer 3,5-mm-T-Abstützplatte ungeeignet. Mit der Neu-Entwicklung der winkelstabilen 3,5-mm-T-Abstützplatten am distalen Radius, die von palmar sicher angelegt werden können, werden heutzutage auch nach dorsal dislozierte Frakturtypen (A 3, C 1) sicher von palmar versorgt. Zur anatomie-gerechten Einrichtung und Fixation der 3 Säulen des distalen Radius wird in selteneren Fällen die dorsale Plattenosteosynthese durch $2,4 \mathrm{~mm}$ winkelstabile Platten von radio-dorsal und dorso-ulnar durchgeführt.

Osteoporotisches Knochengewebe erschwert die sichere Schraubenfixation, weshalb hier winkelstabile Implantate verwendet werden sollten. Alternativ hierzu kann die Knochendefektauffüllung mittels Knochenersatzmaterialien erfolgen.

\section{Literatur}

1. Beyermann K, Prommersberger KJ. Die gleichzeitige Versorgung mehrfragmentärer distaler Radiusfrakturen von einem palmaren und dorsalen Zugang. Handchir Mikrochir Plast Chir 2000; 32: 404-410

2. Peine R, Rikli DA et al. Comparison of three different plating techniques for the dorsum of the distal radius: Biomechanical study. Hand Surg (Am) 2000; 25: 29-33

3. Rikli DA, Regazzoni T. The double plating technique for distal radius fractures. Techniques in Hand and Upper Extremities Surgery 2000; 4: 107-114

4. Siebert HR. Distale Radiusfrakturen an typischer Stelle, Behandlungsverfahren. Aktuelle Traumatologie 1997; 27: 7-15

Prof. Dr. med. Hartmut R. Siebert Chefarzt

Chirurgische Klinik II

Unfall-, Hand- und Wieder-

herstellungschirurgie

Diakonie-Krankenhaus

Diakoniestr. 10

D-74523 Schwäbisch Hall 